\title{
Congenital muscular dystrophy with intellectual disability and severe epilepsy
}

INSERM

\section{Source}

INSERM. (1999). Orphanet: an online rare disease and orphan drug data base. Congenital muscular dystrophy with intellectual disability and severe epilepsy. ORPHA:329178

Congenital muscular dystrophy with intellectual disability and severe epilepsy is a rare, fatal, inborn error of metabolism disorder characterized by respiratory distress and severe hypotonia at birth, severe global developmental delay, early-onset intractable seizures, myopathic fascies with craniofacial dysmorphism (trigonocephaly/progressive microcephaly, low anterior hairline, arched eyebrows, hypotelorism, strabismus, small nose, prominent philtrum, thin upper lip, high-arched palate, micrognathia, malocclusion), severe, congenital flexion joint contractures and elevated serum creatine kinase levels. Scoliosis, optic atrophy, mild hepatomegaly, and hypoplastic genitalia may also be associated. 